\title{
Magnetic and Structural Properties of Fe-Based Nanoparticles
}

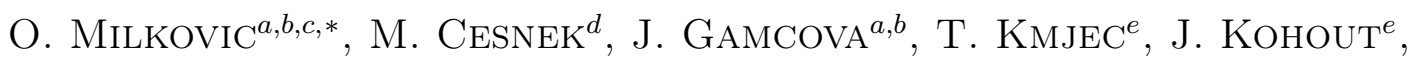 \\ M. REIFFERS ${ }^{f}$ AND R. VARGA ${ }^{g}$ \\ ${ }^{a}$ Institute of Material Research, Slovak Academy of Sciences, Watsonova 47, 04001 Košice, Slovak Republic \\ ${ }^{b}$ Institute of Materials and Quality Engineering, Faculty of Materials, Metallurgy and Recycling, \\ Technical University of Košice, Letná 9, 04200 Košice, Slovak Republic \\ ${ }^{c}$ Institute of Experimental Physics, Slovak Academy of Sciences, Watsonova 47, 04001 Košice, Slovak Republic \\ ${ }^{d}$ Department of Nuclear Reactors, Faculty of Nuclear Sciences and Physical Engineering, \\ Czech Technical University in Prague, V Holešovičkách 2, 18000 Prague 8, Czech Republic \\ ${ }^{e}$ Faculty of Mathematics and Physics, Charles University, V Holešovičkách 2, 18000 Prague, Czech Republic \\ ${ }^{f}$ Faculty of Humanities and Natural Sciences, Prešov University, Prešov, Slovak Republic \\ ${ }^{g}$ CPM-TIP, P.J. Šafárik University, Park Angelinum 9, Košice, 041 54, Slovakia
}

\begin{abstract}
In this study, nanoparticles were prepared by precipitation from solid solution of saturated binary $\mathrm{Cu}-\mathrm{Fe}$ alloy with subsequent electrochemical dissolution of the surrounding matrix. As-prepared nanoparticles were studied by the Mössbauer spectroscopy and scanning transmission electron microscopy. Results show that the as-prepared nanoparticles have core-shell character and their mean size is dependent on annealing time. The Mössbauer spectroscopy revealed major crystalline phase which was identified as $\alpha$-Fe and relatively disordered minor phase which was identified as maghemite $\left(\gamma-\mathrm{Fe}_{2} \mathrm{O}_{3}\right)$. Magnetic measurements suggest that saturated magnetization is not only dependent on core diameter of nanoparticles but also on its structure.
\end{abstract}

DOI: 10.12693/APhysPolA.137.723

PACS/topics: Fe-based nanoparticles, magnetization, Mössbauer spectroscopy

\section{Introduction}

Nanoparticles have very high application potential in industry or medicine due to their unique size and properties [1, 2]. Many scientific groups have recently focused on nanoparticles with superior magnetic properties. Such nanoparticles might be possibly used in medicine as drug carriers, contrast agents for MRI, or in magnetic hyperthermia treatment $[3,4]$. One of the problems in this field is biocompatibility of nanoparticles. Except of medicine, such magnetic nanoparticles might be also used for environmental remediation and many other applications [5, 6]. Among many others, Fe-based nanoparticles exhibit relatively good magnetic properties and good biocompatibility [7]. One of the groups of Fe-based nanoparticles are so called zerovalent iron nanoparticles. As it was reported earlier iron nanoparticles might have some advantages over the other particles mainly due to their relatively high magnetic moment [8]. This paper focuses on magnetic and structural properties of Fe-based nanoparticles prepared by precipitation from solid solution of saturated binary $\mathrm{Cu}-\mathrm{Fe}$ alloy.

\section{Samples and methods}

Samples of Fe-based nanoparticles were prepared by precipitation from solid solution of oversaturated binary

\footnotetext{
*corresponding author; e-mail: omilkovic@saske.sk
}

Labeling samples and basic parameters.

TABLE I

\begin{tabular}{c|c|c|c|c}
\hline \hline Sample & $\begin{array}{c}\text { Anneal. } \\
\text { time [h] }\end{array}$ & $\begin{array}{c}\text { Mean } \\
\text { diameter [nm] }\end{array}$ & $\begin{array}{c}\text { Weight } \\
{[\%]}\end{array}$ & $\begin{array}{c}\text { MS } \\
{\left[\mathrm{A} \mathrm{m}^{2} / \mathrm{kg}\right]}\end{array}$ \\
\hline $\mathrm{A}$ & 3 & 17.5 & 31.9 & 68.4 \\
$\mathrm{~B}$ & 6 & 20.8 & 43.1 & 88.2 \\
$\mathrm{C}$ & 9 & 22 & 45 & 92.5 \\
$\mathrm{D}$ & 12 & 30.1 & 64.5 & 104.2 \\
$\mathrm{E}$ & 18 & 36.5 & 65.7 & 111.9
\end{tabular}

$\mathrm{Cu}-\mathrm{Fe}$ alloy quenched after homogenization annealing at $1273 \mathrm{~K}$ for 18 hours. Such alloy with $1.25 \mathrm{wt} . \%$ of Fe was annealed at $973 \mathrm{~K}$ for different time periods ranging from 3 to $18 \mathrm{~h}$ in argon atmosphere in order to obtain the precipitates with different mean size. The $\mathrm{Cu}$ matrix was subsequently dissolved with help of $\mathrm{NH}_{4} \mathrm{OH}+\mathrm{H}_{2} \mathrm{O}_{2}$ solution. Labeling samples and basic parameters are listed in Table I.

Shape and size distribution of as prepared nanoparticles were analyzed by scanning transmission electron microscopy (TEM, JEOL-2100F) dropped on carbon replicas.

Structure of the samples was analyzed by ${ }^{57} \mathrm{Fe}$ Mössbauer spectroscopy (MS) which can also provide a valuable information about magnetic ordering. All of the MS spectra were recorded in transmission mode using a constant acceleration spectrometer with a ${ }^{57} \mathrm{Co} / \mathrm{Rh}$ source. Spectra were recorded at $4.2 \mathrm{~K}$ using helium bath cryostat. Resulting isomer shifts are quoted relative to the MS spectrum of $12.5 \mu \mathrm{m}$ thick $b c c$-Fe foil obtained at room temperature $(R T)$. Isomer shift (IS), hyperfine 
magnetic field $\left(B_{h f}\right)$, line width $(\Gamma)$, and area of spectral components $(A)$ were refined by the curve fitting software CONFIT [9].

Magnetic measurements were carried out using the Vibrating Sample Magnetometer (VSM) on DYNACOOL device of Quantum design. Temperature dependencies of magnetization were performed in zero field cooled (ZFC) and field cooled (FC) mode with applied magnetic field of $10 \mathrm{kOe}$.

\section{Results and discussion}

Figure 1 shows STEM images of as-prepared nanoparticles. Particles are close to spherical morphology and with the evident core-shell structure. Previous results obtained by electron diffraction of such nanoparticles revealed $\alpha$-Fe in the core and iron oxide in the shell of studied nanoparticles [10, 11].

Figure 2 shows MS spectra recorded at temperature $4.2 \mathrm{~K}$. All the spectra exhibit very similar six-line patterns characteristic for magnetically ordered materials. Major sextet with relativelly narrow lines was identified as $\alpha$-Fe (IS $\sim 0.13-0.14 \mathrm{~mm} / \mathrm{s}$, and $B_{h f} \sim 33.9-34.5 \mathrm{~T}$ ). Relatively broad minor six-line pattern was fitted with model consisting of two distributions of hyperfine magnetic field. The broadened character of this part of the spectra might suggest disordered or non-well ordered phase. Obtained hyperfine parameters (IS $\sim 0.47-0.50 \mathrm{~mm} / \mathrm{s}, B_{h f} \sim 50.4-51.0 \mathrm{~T}$, and IS $\sim 0.40-0.48 \mathrm{~mm} / \mathrm{s}, B_{h f} \sim 46.4-47.2 \mathrm{~T}$ ) are similar to hyperfine parameters of $\gamma-\mathrm{Fe}_{2} \mathrm{O}_{3}$. Such iron oxide layer was probably created on top of the $\alpha$-Fe during the dissolution of the $\mathrm{Cu}$ matrix. As the $\mathrm{Cu}$ around the $\mathrm{Fe}$ nanoprecipitates was dissolved they got in the contact with the $\mathrm{NH}_{4} \mathrm{OH}+\mathrm{H}_{2} \mathrm{O}_{2}$ solution and local environment and rapidly oxidized. Thus, as-prepared nanoparticles might consist of $\alpha$-Fe core and most likely $\gamma-\mathrm{Fe}_{2} \mathrm{O}_{3}$ shell.

Obtained ZFC and FC curves are shown in Fig. 3. It should be noted that the studied nanoparticles do not exhibit supperparamagnetic behavior due to their relatively large mean size. However, the value of magnetization is much smaller than value of saturated magnetization of bulk $\alpha$-Fe.

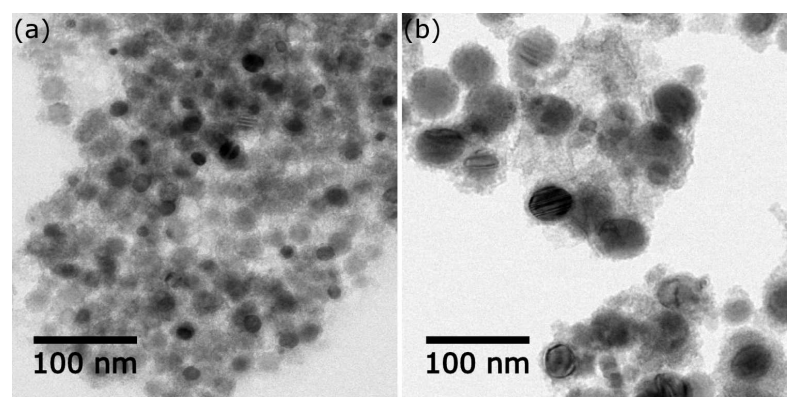

Fig. 1. STEM images of as-prepared nanoparticles with mean diameter (a) $18 \mathrm{~nm}$, and (b) $36 \mathrm{~nm}$.

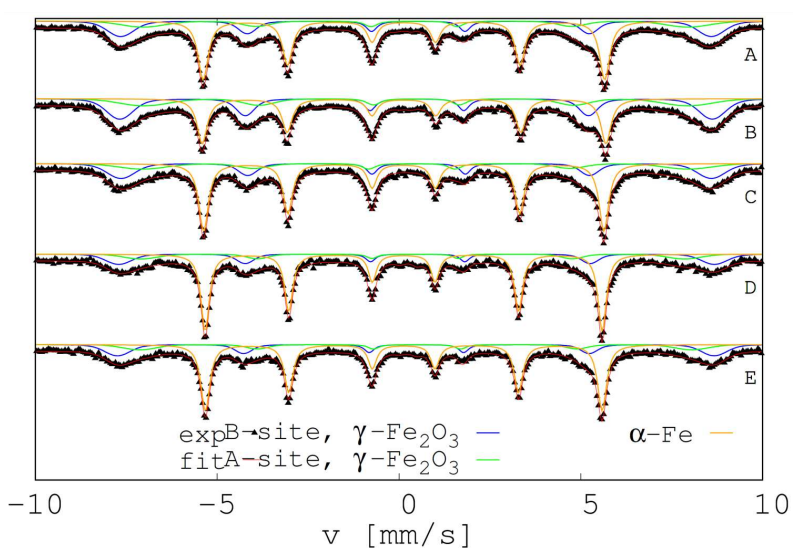

Fig. 2. MS spectra of as-prepared nanoparticles recorded at $4.2 \mathrm{~K}$.

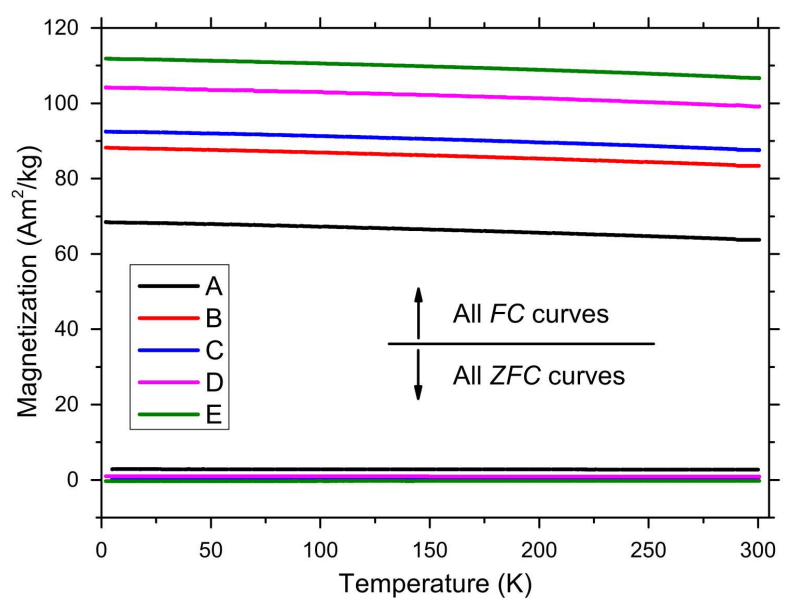

Fig. 3. ZFC and FC curves of nanoparticles. FC mode measured at applied field of $10 \mathrm{kOe}$.

If the weight percentage calculated from XRD and TEM $[11,12]$ of presented phases is taken into account following approximation for the saturated magnetization can be used

$$
M=x M_{\text {core }}+(1-x) M_{\text {shell }},
$$

where $M$ is the overall saturated magnetization of the particles, $M_{\text {core }}$ is the saturated magnetization of the particle core, $M_{\text {shell }}$ is the saturated magnetization of the particle shell, and $x$ is volume ratio of the particle core. Figure 4 shows saturated magnetization at $5 \mathrm{~K}$ as a function of iron core diameter (black line) together with calculated values of saturated magnetization using standard bulk values of $\alpha$-Fe $\left(218 \mathrm{Am}^{2} / \mathrm{kg}\right)$ and $\gamma-\mathrm{Fe}_{2} \mathrm{O}_{3}$ $\left(35 \mathrm{Am}^{2} / \mathrm{kg}\right.$ ) (red line). It can be seen that value of calculated magnetization is higher in contrast to measured one. Furthermore, one can notice a gap between measured and calculated values. This gap also increases with increasing iron core size. Such gap between the values might be observed probably due to the effect of twinning and width of size distribution. The concentration of mentioned twinned crystals and the width of particle 


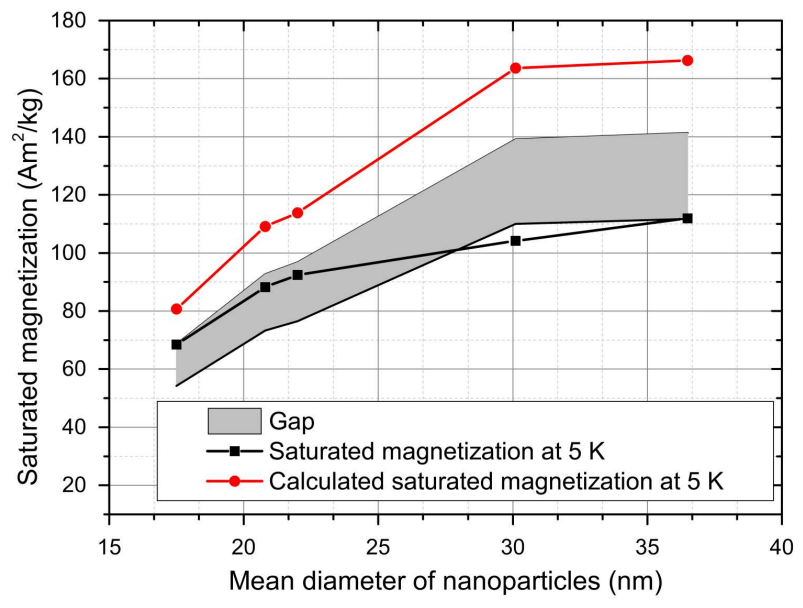

Fig. 4. Dependence of measured saturated magnetization at $5 \mathrm{~K}$ (black line), calculated saturated magnetization using value $218 \mathrm{~A} \mathrm{~m}^{2} / \mathrm{kg}$ for $\alpha$-Fe (red line), and gap of calculated saturated magnetization using values for $\alpha$-Fe core between 135 and $180 \mathrm{~A} \mathrm{~m}^{2} / \mathrm{kg}$ (grey area) on diameter of nanoparticles.

size distribution increases with increasing size of the iron core. Thus, the gap between values grows. It was also reported earlier that the crystallinity affects magnetization of small volume objects as nanoparticles [13].

\section{Conclusions}

Fe-based nanoparticles prepared by precipitation from solid solution of saturated binary $\mathrm{Cu}-\mathrm{Fe}$ alloy were studied by transmission electron microscopy (TEM), Mössbauer spectroscopy (MS), and magnetic analytical methods. TEM images revealed core-shell and spherical character of as-prepared nanoparticles. MS results revealed minor crystalline phase identified as $\alpha$-Fe which belongs to the core of the nanoparticles and minor disordered phase identified as $\gamma-\mathrm{Fe}_{2} \mathrm{O}_{3}$ which belongs to the shell of the nanoparticles. Results obtained by magnetic analytical methods suggest that saturated magnetization is not only dependent on core diameter of the nanoparticles but also on its structure.

\section{Acknowledgments}

This work was supported by the Scientific Grant Agency VEGA project No. 2/0141/19, grant SGS17/140/OHK4/2T/14, and by The Czech Science Foundation (project No. 19-02584S).

\section{References}

[1] W.J. Stark, P.R. Stoessel, W. Wohlleben, A. Hafner, R. Soc. Chem. 44, 5793 (2015).

[2] O.V. Salata, J. Nanobiotechnol. 2, 3 (2004).

[3] T.A. Esquivel-Castro, M.C. Ibarra-Alonso, J. Oliva, A. Martínez-Luévanos, Mater. Sci. Eng. C 96, 915 (2019).

[4] M. O'Donnell, Physica C 548, 103 (2018).

[5] T.E. Sutto, Environm. Pollut. 243, 528 (2018).

[6] A.A. Kajani, A. Bordbar, J. Hazard. Mater. 366, 268 (2019).

[7] H. Fatima, K. Kim, Adv. Powder Technol. 29, 2678 (2018).

[8] Y. Qiang, J. Antony, A. Sharma, J. Nutting, D. Sikes, D. Meyer, J. Nanopart. Res. 8, 489 (2006).

[9] T. Zak, Y. Jiraskova, Surf. Interface Anal. 38, 710 (2006).

[10] O. Milkovič, G. Janák, Š. Nižník, S. Longauer, L. Fröhlich, Mater. Lett. 64, 144 (2010).

[11] O. Milkovič, J. Gamcová, M. Sopko, I. Škorvánek, Acta Phys. Pol. A 131, 747 (2017).

[12] O. Milkovič, J. Michaliková, J. Bednarčík, Š. Michalik, Key Engineering Materials 662, 217 (2015).

[13] M. Kin, M. Tanaka, Y. Hayashi, J. Hasaegawa, T. Ogawa, J. Appl. Phys. 117, 17E714 (2015). 